Preprint JINR E2-92-337

\title{
TWISTOR-LIKE TYPE II SUPERSTRING AND BOSONIC STRING
}

\author{
V. Chikalov \\ Institute of Applied Physics \\ 244007 Sumy, Ukraine \\ and \\ A. Pashnev \\ JINR-Laboratory of Theoretical Physics \\ Dubna, Head Post Office, P.O.Box 79, 101000 Moscow, Russia
}

\begin{abstract}
The superfield formulation of type II Green-Schwarz superstring with $n=(1,0)$ worldsheet supersymmetry is constructed. It is shown that the inclusion of the second spinor coordinate in the target superspase leads to the possibility of the reparametrization invariant description of the superstring in the absence of any field from the two dimensional supergravity multiplet. The twistor-like action of Chern-Simons type for bosonic string in $D=3,4,6,10$ is deduced from the superstring action.
\end{abstract}

Submitted to Mod. Phys. Lett. A

*BITNET: PASHNEV@THEOR.JINRC.DUBNA.SU 


\section{Introduction}

In spite of the importance of the well-known $\kappa$-symmetry of superparticle and superstring Lagrangians, its nature was not completely investigated up to now. Therefore the idea of the reformulation of superparticle and superstring Lagrangians in which this symmetry has clear geometrical meaning is very attractive and a great deal of attention has been devoted to this problem [3]- 16].

For the first time this idea was realized by Sorokin, Tkach and Volkov (STV) in the paper [3] in which the twistor-like formulations for $D=3,4$ superparticle with local $n=1,2$ superconformal invariance were proposed. They further showed that the fermionic $\kappa$-symmetry of the conventional Brink-Schwarz superparticle is closely related to the local superconformal transformations in the corresponding superworldline. The idea of double Grassmann analiticity in proper and target superspaces was formulated and twistor-like actions for $D=3,4,6$ superparticle with $n=1,2,4$ superconformal worldline symmetry was constructed in [5] (including nontrivial background).

There has been previous work on the construction of the twistor- like action for the $D=10, n=8$ superparticle [9, 8] and, as it was shown by Galperin and Sokatchev [1], double analiticity is not necessary and they constructed an action which has similar form for all dimensions $D=3,4,6,10$ with $n=1,2,4,8$. This action looks like a strightforward generalization of (STV) action for $D=3 n=1$ case [3].

In all examples considered the $\kappa$-symmetry of the superparticle was connected with the local superconformal transformations which form the subgroup of the group of superdiffeomorphism of corresponding superworldline. Preliminary investigations show that in the case of superstring the situation is analogous. First steps in constructing the twistor-like form of Green-Schwarz superstring [11] have been made in superconformal gauge [12]-[13] and the problem of finding the reparametrization invariant action was formulated. Partially this problem was solved for heterotic superstring in different approaches 14. 16. However, all of the actions in these papers contain some of the fields of two dimensional supergravity multiplet to ensure the reparametrization invariance of the action. If there are no additional fields, as in the paper by Ivanov and Kapustnikov [6], only one of two Virasoro conditions is fulfilled and part of the reparametrization transformations is broken. In [16] it was shown how to include one additional field from two dimensional supergravity multiplet to restore the reparametrization invariance and twistor-like action with $n=(4,0)$ worldsheet supersymmetry in $D=6$ was constructed.

In this paper we construct the twistor-like action for type II Green-Schwarz superstring [11] with $n=(1,0)$ superworldsheet , parametrized by two bosonic (time-like and space-like) and one Grassmann coordinates. The inclusion of second spinor Grassmann superfield, which is necessary to describe type II superstring, leads to rather nontrivial consequence: in spite of the absence of fields from supergravity multiplet, the action is invariant under the general reparametrizations of the superworldsheet .

In the second part of the paper we consider simpler case of the superparticle in the formulation of Sorokin, Tkach and Volkov [3] and Galperin and Sokatchev[11]. We show in what sense this action is invariant not only under the superconformal transformations, but under the general reparametrizations of the $n$-extended superworldline as well. The action for superstring and transformation laws for all superfields under general 
reparametrizations of the superworldsheet are constructed in the third part of the work.

At the end of the paper we briefly discuss the twistor-like action for bosonic string which is the part of the component action for type II superstring. It has very simple form including only bilinear and trilinear couplings and do not contain metric fields. Nevertheless,in $D=3,4,6,10$ this action is invariant under the transformations of twodimensional diffeomorphism group of the string worldsheet.

\section{The superparticle and its symmetry group}

In this section we briefly describe the Galperin-Sokatchev [11] twistor-like formulation of $D=10$ superparticle action with manifest $n=8$ world-line supersymmetry and show that the symmetry group of this action actually includes the arbitrary reparametrizations of the $(1,8)$ world-line superspace with 1 time-like even coordinate $\tau$ and 8 odd coordinates $\eta^{a}$. The Galperin-Sokachev action for $D=10$ superparticle

$$
S=\int d \tau d^{8} \eta P_{a \mu}\left(D_{a} X^{\mu}-i D_{a} \Theta \gamma^{\mu} \Theta\right)=\int d \tau d^{8} \eta P_{a \mu} \Omega_{a}^{\mu}
$$

is an integral over the $n=8$ world-line superspace and describes the dynamics of $n=8$ superfields $X(\tau, \eta)$ and $\Theta(\tau, \eta)$ which are correspondingly the commuting vector and anticommuting Majorana-Weil spinor coordinates of 10-dimensional target $n=1$ superspace. The Lagrange multiplier $P_{a \mu}$ is anticommuting 10-dimensional vector and carries also a world-line $O(8)$ index $a$. The definition of $n=8$ supercovariant derivatives $D_{a}$ is as follows:

$$
D_{a}=\frac{\partial}{\partial \eta_{a}}+i \eta_{a} \frac{\partial}{\partial \tau}, \quad\left\{D_{a}, D_{b}\right\}=2 i \delta_{a b} \frac{\partial}{\partial \tau} .
$$

As was shown in [11], the action (2.1) is invariant under global $N=1, D=10$ targetspace supersymmetry and under the $n=8$ world-line superconformal group which is the subgroup of the general superdiffeomorphism group of the $(1,8)$ world-line superspace.

In spite of the fact that the action (2.1) is written in terms of the flat supercovariant derivatives it is invariant under the arbitrary reparametrization of the space $\tau, \eta_{a}$. This miraculous fact for any $n$ is based on the following simple arguments.

The variation of the flat world-line supercovariant derivatives (2.1) under the general infinitesimal transformation of world-line coordinates

$$
\tau \rightarrow \tau+\alpha(\tau, \eta), \eta_{a} \rightarrow \eta_{a}+\xi_{a}(\tau, \eta)
$$

is nonhomogenious in the $D_{a}$ and contains the term with ordinary time derivative

$$
\delta D_{a}=-\left(D_{a} \xi_{b}\right) D_{b}+2 i \zeta_{a} \frac{\partial}{\partial \tau}
$$

where

$$
\zeta_{a}=\xi_{a}+\frac{i}{2} D_{a} \Lambda, \quad \Lambda \equiv \alpha+i \eta_{a} \xi_{a}
$$

Only in the case of superconformal transformations for which $\zeta_{a}=0$ the last term in (2.4) disappears. However this term is not so dangerous. To see this let us calculate the 
variation of the quantity $\Omega_{a}^{\mu}$ in $(2.1)$. The total variation of this quantity consists of two parts

$$
\delta \Omega_{a}^{\mu}=\delta^{1} \Omega_{a}^{\mu}+\delta^{2} \Omega_{a}^{\mu}
$$

first of which is due to variation (2.4) of flat supercovariant derivatives (2.2) and second is due to variations of dynamical superfields $\delta X^{\mu} \equiv X^{\prime \mu}\left(\tau^{\prime}, \eta^{\prime}\right)-X^{\mu}(\tau, \eta)$ etc. if we assume that these fields are not scalars under the general coordinate transformations (2.3) . Simple calculations lead to

$$
\delta^{1} \Omega_{a}^{\mu}=-\left(D_{a} \xi_{b}\right) \Omega_{a}^{\mu}+2 i \zeta_{a}\left(\dot{X}^{\mu}-i \dot{\Theta} \gamma^{\mu} \Theta\right)
$$

where $\dot{X}^{\mu} \equiv \frac{\partial}{\partial \tau} X^{\mu}$. Using the identity

$$
D_{b} \Omega_{a}^{\mu}+D_{a} \Omega_{b}^{\mu}=-2 i D_{a} \Theta \gamma^{\mu} D_{b} \Theta+2 i \delta_{a b}\left(\dot{X}^{\mu}-i \dot{\Theta} \gamma^{\mu} \Theta\right)
$$

which is due to the commutation relations (2.2) and assuming the following transformation laws for $X^{\mu}$ and $\Theta$

$$
\begin{aligned}
\delta X^{\mu} & =\zeta_{a} D_{a} X^{\mu} \\
\delta \Theta & =\zeta_{a} D_{a} \Theta
\end{aligned}
$$

we find the total variation of the $\Omega_{a}^{\mu}$

$$
\delta \Omega_{a}^{\mu}=\frac{i}{2}\left(D_{a} D_{b} \Lambda\right) \Omega_{b}^{\mu}+\zeta_{b} D_{b} \Omega_{a}^{\mu}
$$

The linearity of this expression in $\Omega_{a}^{\mu}$ and $D_{b} \Omega_{a}^{\mu}$ is crucial for invariance of the action (2.1). Taking into account the variation of the supervolume element

$$
\delta\left(d \tau d^{n} \eta\right)=\left(\frac{\partial}{\partial \tau} \alpha-\frac{\partial}{\partial \eta_{a}} \xi_{a}\right)\left(d \tau d^{n} \eta\right)=\left(\dot{\Lambda}-D_{a} \xi_{a}\right)\left(d \tau d^{n} \eta\right)
$$

and integrating by parts we find the following expression for $\delta S$ :

$$
\begin{aligned}
\delta S= & \int d \tau d^{n} \eta\left\{\left(\delta P_{a \mu}-\zeta_{b} D_{b} P_{a \mu}-\frac{n-1}{2} \dot{\Lambda} P_{a \mu}-\right.\right. \\
& \left.\left.-\frac{i}{4}\left(\left[D_{a}, D_{b}\right] \Lambda\right) P_{b \mu}\right) \Omega_{a}^{\mu}--D_{b}\left(\zeta_{b} P_{a \mu} \Omega_{a}^{\mu}\right)\right\}
\end{aligned}
$$

Since the last term in the equation (2.12) is a total derivative, the action is invariant if

$$
\delta P_{a \mu}=\frac{n-1}{2} \dot{\Lambda} P_{a \mu}+\frac{i}{4}\left(\left[D_{a}, D_{b}\right] \Lambda\right) P_{b \mu}+\zeta_{b} D_{b} P_{a \mu}
$$

Thus the action (2.1) for any $n$ is also invariant under the arbitrary reparametrizations of the $(1, n)$ superworldline. The transformation laws (2.8) show that superfields $X^{\mu}(\tau, \eta)$ and $\Theta(\tau, \eta)$ which behaves as scalars under the transformations of superconformal group lose this property for transformations (2.3) with $\zeta_{a}(\tau, \eta) \neq 0$. The transformation law for $P_{a \mu}$ (see [11]) is also modified by the last term in eq. 2.13) having the structure 
analogous to that in the transformation laws for all other fields. The Chern-Simons nature of the action (2.1) [4], i.e. the invariance of the action under the general coordinate transformations in the absence of one-dimensional supergravity multiplet is confirmed.

The interesting feature of the transformation laws (2.8),(2.13) is that in the active form (we consider for simplicity only $X^{\mu}$ )

$$
\begin{aligned}
\bar{\delta} X^{\mu} & \equiv \delta X_{\mu}-\xi_{a} \frac{\partial}{\partial \eta_{a}} X^{\mu}-\alpha \frac{\partial}{\partial \tau} X^{\mu} \\
& =-\left(\Lambda-\frac{1}{2} \eta_{a} D_{a} \Lambda\right) \frac{\partial X^{\mu}}{\partial \tau}+\frac{i}{2} D_{a} \Lambda \frac{\partial X^{\mu}}{\partial \eta_{a}}
\end{aligned}
$$

they have the form of superconformal transformations of the scalar superfield [11](appropriately modified for $\left.P_{a \mu}\right)$ with parameter $\Lambda(\tau, \eta)$ constructed from arbitrary parameters $\alpha(\tau, \eta)$ and $\xi_{a}(\tau, \eta)$ according to the eq. (2.4). Thus, all the superfields considered demonstrate some sort of mimicry, i.e. their transformation laws (2.8),(2.13) for general reparametrizations (2.3) of superworldsheet are adapted in such a way, that only particular combination $\Lambda$ of transformation parameters makes real contribution to their active form of transformation laws. Analogous effect, as we will see, takes place in the twistorlike formulation of superstring .

\section{$3 n=(1,0)$ Superstring in $N=2$ extended target su- perspace}

The superworldsheet of the superstring is parametrized by two bosonic $\tau^{0}, \tau^{1}$ and one fermionic $\eta$ coordinates. We first introduce superfields

$$
\begin{aligned}
X^{\mu}\left(\tau^{i}, \eta\right) & =x^{\mu}\left(\tau^{i}\right)+i \eta \chi^{\mu}\left(\tau^{i}\right) \\
P_{\mu}\left(\tau^{i}, \eta\right) & =p_{\mu}\left(\tau^{i}\right)+i \eta \rho\left(\tau^{i}\right), \\
\Theta^{A}\left(\tau^{i}, \eta\right) & =\theta^{A}\left(\tau^{i}\right)+\eta \lambda^{A}\left(\tau^{i}\right), \quad A=1,2
\end{aligned}
$$

and flat covariant derivative

$$
D=\frac{\partial}{\partial \eta}+i \eta \frac{\partial}{\partial \tau^{0}}, \quad D^{2}=i \frac{\partial}{\partial \tau^{0}} .
$$

in the superspace $\left(\tau_{0}, \eta\right)$.

We propose the following action for the superstring

$$
\begin{aligned}
S= & \int d^{2} \tau d \eta\left\{\left(-i P_{\mu}+\Theta^{1} \gamma_{\mu} \Theta^{1^{\prime}}-\Theta^{2} \gamma_{\mu} \Theta^{2^{\prime}}\right) \Omega_{0}^{\mu}\right. \\
+ & \left(D \Theta^{1} \gamma_{\mu} \Theta^{1}-D \Theta^{2} \gamma_{\mu} \Theta^{2}\right) \Omega_{1}^{\mu}- \\
& \left.\quad-i D \Theta^{1} \gamma_{\mu} \Theta^{1} \Theta^{2} \gamma^{\mu} \Theta^{2^{\prime}}+i \Theta^{1} \gamma_{\mu} \Theta^{1^{\prime}} D \Theta^{2} \gamma^{\mu} \Theta^{2}\right\}
\end{aligned}
$$

where

$$
\begin{aligned}
\Omega_{0}^{\mu}= & D X^{\mu}-i D \Theta^{1} \gamma^{\mu} \Theta^{1}-i D \Theta^{2} \gamma^{\mu} \Theta^{2} \\
\Omega_{1}^{\mu}= & X^{\mu^{\prime}}-i \Theta^{1^{\prime}} \gamma^{\mu} \Theta^{1}-i \Theta^{2^{\prime}} \gamma^{\mu} \Theta^{2} \\
& \Theta^{A^{\prime}} \equiv \frac{\partial}{\partial \tau^{1}} \Theta^{A}
\end{aligned}
$$


The action (3.5) is invariant under the transformations of extended $N=2$ supersymmetry in target superspace $\left(X^{\mu}, \Theta^{A}\right)$

$$
\delta \Theta^{A}=\epsilon^{A}, \quad \delta X^{\mu}=i \Theta^{A} \gamma^{\mu} \epsilon^{A}, \quad \delta P_{\mu}=0
$$

in $D=3,4,6$ and 10 where the relation

$$
\left(\gamma^{\mu}\right)_{(\alpha \beta}\left(\gamma_{\mu}\right)_{\gamma) \delta}=0
$$

takes place. When the target superspace is not extended $(N=1)$, i.e. $\Theta^{2}=0$, the quartic in $\Theta$ 's terms disappear and to within the redefinition of Lagrange multiplier $P_{\mu}$ our action coinsides with the action for the $n=(1,0)$ twistorlike superstring proposed in the paper [16] (see also [6]). Such an extension of the target superspace leads to highly nontrivial consequences. namely, the action (3.5) is invariant, as we will see later, under the general reparametrizations of superworldsheet $\left(\tau^{i}, \eta\right)$ without introducing any additional fields such as supergravity multiplet. Due to this invariance we can use usual (not lightlike) notations $\tau^{0}, \tau^{1}$ for bosonic coordinates of superworldsheet .

The most general coordinate transformation of the superworldsheet $\left(\tau^{i}, \eta\right)$ have the form:

$$
\delta \tau^{i}=\alpha^{i}(\tau, \eta), \quad \delta \eta=\xi(\tau, \eta)
$$

and the following combinations of these parameters

$$
\Lambda=\alpha^{0}+i \eta \xi, \quad \zeta=\frac{i}{2} D \Lambda+\xi
$$

will be useful as well. The transformation laws for derivatives and for the integration measure are:

$$
\begin{aligned}
\delta D & =-\left(D \alpha^{1}\right) \frac{\partial}{\partial \tau^{1}}-(D \xi) D+2 \zeta D^{2} \\
\delta \frac{\partial}{\partial \tau^{1}} & =-\alpha^{1^{\prime}} \frac{\partial}{\partial \tau^{1}}-\xi^{\prime} D+i \Lambda^{\prime} D^{2} \\
\delta\left(d^{2} \tau d \eta\right) & =\left(\dot{\alpha}^{0}+\alpha^{1^{\prime}}-\frac{\partial \xi}{\partial \eta}\right) d^{2} \tau d \eta .
\end{aligned}
$$

If the superfields $X^{\mu}, \Theta^{A}$ transform under the 3.10 in the following way

$$
\begin{aligned}
\delta X^{\mu}= & \zeta D X^{\mu}+\frac{D \alpha^{1}}{2 R^{2}}\left(\Theta^{1} \gamma^{\mu} \hat{P} \hat{R} D \Theta^{1}+\Theta^{2} \gamma^{\mu} \hat{P} \hat{R} D \Theta^{2}\right) \\
\delta \Theta^{A}= & \zeta D \Theta^{A}-i \frac{D \alpha^{1}}{2 R^{2}} \hat{P} \hat{R} D \Theta^{A} \\
R_{\mu}= & D \Theta^{1} \gamma_{\mu} D \Theta^{1}-D \Theta^{2} \gamma_{\mu} D \Theta^{2} \\
\delta P_{\mu}= & \zeta D P_{\mu}-\frac{D \alpha^{1}}{R^{2}}\left(\Theta^{1^{\prime}} \gamma_{\mu} \hat{P} \hat{R} D \Theta^{1}-\Theta^{2^{\prime}} \gamma_{\mu} \hat{P} \hat{R} D \Theta^{2}\right)- \\
& \quad-\alpha^{1^{\prime}} P_{\mu}+\Lambda^{\prime} R_{\mu}
\end{aligned}
$$

where $\hat{P} \equiv P_{\mu} \gamma^{\mu}$, the integrand in (3.5) is invariant up to a total derivatives in critical dimensions $D=3,4,6,10$. One can easily see that only two superfields $\Lambda$ and $\alpha^{1}$ contribute to the active form of these transformations in the whole analogy with the case of superparticle. 
To see that action (3.5) indeed describes the superstring we calculate the component form of the action. After performing the $\eta$ - integration we obtain

$$
\begin{aligned}
S= & \int d^{2} \tau\left\{p_{\mu}\left(\omega_{0}^{\mu}-\lambda^{1} \gamma^{\mu} \lambda^{1}-\lambda^{2} \gamma^{\mu} \lambda^{2}\right)+\omega_{1 \mu}\left(\lambda^{1} \gamma^{\mu} \lambda^{1}-\lambda^{2} \gamma^{\mu} \lambda^{2}\right)+\right. \\
& +i \dot{x}^{\mu}\left(\theta^{1} \gamma_{\mu} \theta^{1^{\prime}}-\theta^{2} \gamma_{\mu} \theta^{2^{\prime}}\right)-i x^{\mu \prime}\left(\theta^{1} \gamma_{\mu} \dot{\theta}^{1}-\theta^{2} \gamma_{\mu} \dot{\theta}^{2}\right)+ \\
& +\theta^{1} \gamma_{\mu} \dot{\theta}^{1} \theta^{2} \gamma^{\mu} \theta^{2^{\prime}}-\theta^{1} \gamma_{\mu} \theta^{1^{\prime}} \theta^{2} \gamma^{\mu} \dot{\theta}^{2}+ \\
& +i\left(\rho_{\mu}+2 \lambda^{1} \gamma_{\mu} \theta^{1^{\prime}}-2 \lambda^{2} \gamma_{\mu} \theta^{2^{\prime}}\right)\left(\chi^{\mu}-\lambda^{1} \gamma^{\mu} \theta^{1}-\lambda^{2} \gamma^{\mu} \theta^{2}\right)+ \\
& \left.+i\left(\chi^{\mu}\left(\lambda^{1} \gamma_{\mu} \theta^{1}-\lambda^{2} \gamma_{\mu} \theta^{2}\right)\right)^{\prime}+i\left(\lambda^{1} \gamma^{\mu} \theta^{1} \lambda^{2} \gamma_{\mu} \theta^{2}\right)^{\prime}\right\}
\end{aligned}
$$

where

$$
\omega_{0}^{\mu}=\dot{x}^{\mu}-i \dot{\theta}^{1} \gamma^{\mu} \theta^{1}-i \dot{\theta}^{2} \gamma^{\mu} \theta^{2}, \omega_{1}^{\mu}=x^{\mu \prime}-i \theta^{1^{\prime}} \gamma^{\mu} \theta^{1}-i \theta^{2} \gamma^{\mu} \theta^{2}
$$

We first analize the structure of the action and investigate equations of motion. The last two terms in the action (3.19) are total derivatives and they give no contribution to the equations of motion. Only the third term from the end of the action contains fields $\rho_{\mu}$ and $\chi^{\mu}$. These fields are auxiliary because their equations of motion simply express them in terms of all other fields. The most fundamental role in the action (3.19) is played by bosonic fields $\lambda^{A}$ which are spinors of the target space. Their equations of motion

$$
\begin{aligned}
& \left(\hat{p}-\hat{\omega}_{1}\right) \lambda^{1}=0 \\
& \left(\hat{p}+\hat{\omega}_{1}\right) \lambda^{2}=0
\end{aligned}
$$

in dimensions $D=3,4,6,10$ lead to the following relations

$$
\begin{aligned}
p_{\mu}-\omega_{1 \mu} & =e_{1}\left(\tau^{i}\right)\left(\lambda^{1} \gamma_{\mu} \lambda^{1}\right) \\
p_{\mu}+\omega_{1 \mu} & =e_{2}\left(\tau^{i}\right)\left(\lambda^{2} \gamma_{\mu} \lambda^{2}\right)
\end{aligned}
$$

with two arbitrary functions $e_{1,2}\left(\tau^{i}\right)$. As a concequence, these relations reproduce two Virasoro conditions

$$
\left(p_{\mu} \pm \omega_{1 \mu}\right)^{2}=0
$$

To construct the Lagrangian in more familiar form we first find expressions $\lambda^{A} \gamma_{\mu} \lambda^{A}$ from (3.23)-(3.24) and substitute them in the Lagrangian (3.19). After elimination of $p_{\mu}$ due to it's equation of motion, we will obtain the standard Lagrangian for superstring [1] in which metric tensor $g^{i k}$ is expressed in terms of two independent functions $e_{1,2}\left(\tau^{i}\right)$. Thus on mass shell the Lagrangian (3.19) is equivalent to the Green-Shwarz Lagrangian[1].

\section{Discussion}

The twistor-like action (3.5) is written in terms of $n=(1,0)$ worldsheet superfields. In spite of the fact that only flat derivatives $D$ and $\partial / \partial \tau^{1}$ enter the formula (3.5), the action is invariant under the general reparametrizations (3.10) of the superworldsheet $\left(\tau^{i}, \eta\right)$. Unlike the previous investigations [6,15-17], we consider the case of $N=2$ extended target superspace which correspond to the type II Green-Schwarz superstring. In the limit when one of the Grassmann superfields is zero, our action coincides with the action of the work 
|16] (see also [6] ). However the presence of second Grassmann superfield makes it possible to wright down transformations for all superfields under the arbitrary reparametrizations of superworldsheet in absence of two dimensional supergravity multiplet.

To make things more simple, let us consider the twistor-like formulation of bosonic string which is trivially obtained from the component action (3.19) for superstring

$$
S_{B}=\int d^{2} \tau\left\{p_{\mu}\left(\dot{x}^{\mu}-\lambda^{1} \gamma^{\mu} \lambda^{1}-\lambda^{2} \gamma^{\mu} \lambda^{2}\right)+x^{\mu \prime}\left(\lambda^{1} \gamma^{\mu} \lambda^{1}-\lambda^{2} \gamma^{\mu} \lambda^{2}\right)\right\}
$$

The classical equivalence of the action (4.1) with the conventional action for bosonic string can be proved along the same line, as it was proved for the superstring in preceding section. The action is written in terms of flat derivatives and contains only bilinear and trilinear couplings. In spite of the absence of any metric fields, this action is reparametrization invariant with the following transformation laws for all fields (in the active form):

$$
\begin{aligned}
\bar{\delta} \lambda^{A} & =-\frac{1}{2} \dot{\alpha}^{0} \lambda^{A}+\frac{\dot{\alpha}^{1}}{2 r^{2}} \hat{p} \hat{r} \lambda^{A}-\alpha^{0} \dot{\lambda}^{A}-\alpha^{1} \lambda^{A^{\prime}} \\
r_{\mu} & =\lambda^{1} \gamma_{\mu} \lambda^{1}-\lambda^{2} \gamma_{\mu} \lambda^{2} \\
\bar{\delta} p_{\mu} & =-\alpha^{1^{\prime}} p_{\mu}+\alpha^{0^{\prime}} r_{\mu}-\alpha^{0} \dot{p}_{\mu}-\alpha^{1} p_{\mu}{ }^{\prime} \\
\bar{\delta} x^{\mu} & =-\alpha^{0} \dot{x}^{\mu}-\alpha^{1} x^{\mu \prime} .
\end{aligned}
$$

These transformation laws are simple consequences of superfield transformation laws (3.15) written in components. The commutator of two transformations (4.2)-(4.5) has the same form

$$
\left[\bar{\delta}_{1}, \bar{\delta}_{2}\right]=\bar{\delta}_{3}
$$

with parameters $\alpha_{3}^{i}$ expressed in terms of $\alpha_{1}^{i}$ and $\alpha_{2}^{i}$ with the help of standard formulas of two dimensional diffeomorphism group:

$$
\alpha_{3}^{i}=\alpha_{1}^{k} \partial_{k} \alpha_{2}^{i}-\alpha_{2}^{k} \partial_{k} \alpha_{1}^{i}
$$

Note, that the closure property (4.6) of transformations (4.2)-(4.5) takes place only in the target spaces with $D=3,4,6$ and 10 in which the relation (3.9) is valid.

Only $x^{\mu}$ transform under the transformations of diffeomorphism group as a scalar. The transformation laws for all other fields are more complicated. In some sense these fields play the role of zweibein to ensure the repapametrization invariance of the action.

In the case of superstring the situation is more complicated. Though the transformations (3.15-3.18) compensate the arbitrary superworldsheet reparametrization (3.10), their commutator in the active form contains some additional transformations which also do not change the superstring action (3.5). The description of the properties of transformations (3.15)-(3.18) in more details will be given elsewhere.

\section{Acknowledgements}

It is a pleasure for us to thanks participants of the seminar "Supersymmetry-92" (1-3 of July 1992 in Dubna near the Moscow) for useful discussions. 


\section{References}

[1] L.Brink and J.H.Schwarz, Phys. Lett.,B 100(1981) 310.

[2] M.B. Green and J.H. Schwarz, Phys. Lett.,B 136(1984) 367.

[3] D.P. Sorokin, V.I. Tkach and D.V. Volkov, Mod. Phys. Lett., A 4(1989) 901.

[4] P.S. Howe and P.K. Townsend, Phys. Lett.,B 259(1991) 285.

[5] F. Delduc and E. Sokatchev, Class. Quant. Grav.,9(1992) 361.

[6] E.A. Ivanov and A.A. Kapustnikov, Phys. Lett., B 267(1991) 175.

[7] J.P. Gauntlett, Phys. Lett.,B 272(1991) 25.

[8] F.Delduc, A.Galperin and E.Sokatchev, Nucl. Phys., B368(1992) 143.

[9] A.S. Galperin, P.S. Howe and K.S. Stelle, Nucl. Phys., B 368(1992) 248.

[10] A. Pashnev and D. Sorokin, "Note on superfield formulations of $\mathrm{D}=2,3,4,6$ and 10 superparticles", Preprint JINR E2-92-27, Dubna, 1992.

[11] A.Galperin and E. Sokatchev, "A twistor-like D=10 superparticle action with manifest $n=8$ world-line supersymmetry", Preprint JHU-TIPAC-920010/BOnn-HE-92-07, Baltimore and Bonn, 1992.

[12] N. Berkovits, Phys. Lett.,B 232(1989) 184.

[13] D.V. Volkov and A.A. Zheltukhin, Nucl. Phys., B 335(1990) 723.

[14] M. Tonin, Phys. Lett.,B 266(1991) 312.

" $\kappa$-Symmetry as World Sheet Supersymmetry in $D=10$ Heterotic Superstrings", Preprint DFPD/91/TH/29, Padova, 1991.

[15] N.Berkovits, "The Heterotic Green-Schwarz Superstring on an $n=(2,0)$ SuperWorldsheet", Stony Brook preprint ITP-SB-91-69.

[16] F. Delduc, E. Ivanov and E. Sokatchev, "Twistor-like superstrings with D = 3, 4, 6 target superspace and $\mathrm{n}=(1,0),(2,0),(4,0)$ world-sheet supersymmetry",Preprint EnSLAPP-L-371/92/BOnn-HE-92-11, Lyon and Bonn, 1992. 